\title{
Using GPS data to monitor road traffic flows in a metropolitan area: methodology and case study
}

\author{
Alfio Costanzo \\ Department of Electrical, Electronics and Computer Engineering, \\ University of Catania, viale Andrea Doria 6, \\ Catania, 95125, Italy \\ e-mail: alfio.costanzo@dieei.unict.it
}

\begin{abstract}
Currently, few traffic management departments offer traffic monitoring services in real time due to the high cost of such car traffic monitoring system. Aim of the paper is the one of proposing a low cost system based on the GPS signals coming from the smart phones of the drivers to build a colored traffic map and to pass such traffic information to a minimum path finder software resident on a mobility information system or on the user mobile to find better paths to destination. Also, the proposed system may support a decision support system resident on the driver mobile to better plan the commuter paths as well as the user commerce activities in the urban area.
\end{abstract}

Keywords-intelligent transport, internet of things, mobile networked applications.

\section{INTRODUCTION}

The main aim of a mobile information system (MIS) is the one of supporting mobility and logistics activities of both drivers and walking people by using both specialized navigators (e.g., Garmin, Tom-Tom) and smart phones. Usually, a MIS helps mobile users in finding the services most suitable for their current task and the best path to reach them starting from the current user position. However, due to the lack of information about the current car traffic, the suggested path could not correspond to the optimal one. Indeed, all the navigators on the market make use of average traffic information and don't take into account accidents, car congestions and weather conditions that may modify substantially the driver behavior and the travel time of the involved roads.

For these reasons, in many metropolitan areas, the traffic management departments make available to the drivers a traffic map where the main roads are colored depending on the current traffic conditions [1]. However, such traffic indications are not used by the current navigators, thus they should be consulted directly by the drivers to decide whether changing the current path to destination. Also, often such indications deal with the whole car traffic flow of all the lanes of a road, whereas different indications should be given for each lane in order to know more precisely the delays of the different car flows passing from a road to the adjacent ones through their intersection.

Since few traffic management departments offer such services due to the cost of a real time car traffic monitoring system, the paper aims at showing how the GPS signals coming from the smart phones of the drivers may be used not only to build a colored traffic map but also to pass such traffic information to a minimum path finder software resident on a MIS to find, if any, a better path to destination, e.g., [2]. In this way, the MIS may support the driver in deciding how to reach the destination with accurate information obtained at a reasonable cost.

Sect. 2 briefly recalls how the road map is identified by our software in such a way that the road intersections are perfectly superimposed to the ones of Google Maps to inform the mobile users with a familiar interface. Also how such road map is updated dynamically to take into account the changing position of the mobile users is briefly discussed. Sect.3 proposes a methodology to estimate the traffic flow for each lane of a road. This methodology is illustrated by some realistic examples that point out how representing the different flows of a road by different colors. Sect.4 discusses a case study to show the feasibility of the methodology to monitor both the urban and metropolitan roads. The former information is relevant mainly for the commercial and business activities of the citizens, the latter for the commuters. Concluding remarks illustrate the advantage of the proposed methodology and future works.

\section{ROAD MAP: INTERSECTIONS AND USER POSITION}

The Google Maps API allows us to compute the intersections between any two roads R1 and R2 by issuing a DirectionsRequest API whose argument is "R1 \& R2", e.g., if we indicate "10th Avenue \& W 22nd Street, New York", Google Maps gives the geo-coordinates of the intersection that is also visualized suddenly on the Maps (see fig.1).

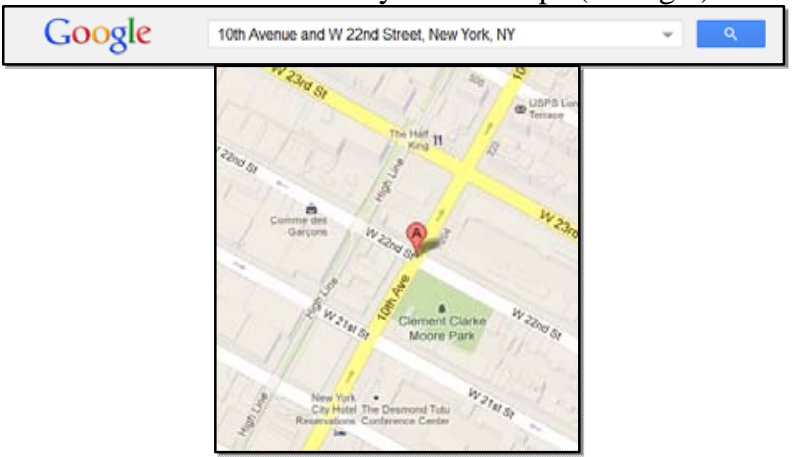

Figure 1. An intersection calculated with Google Maps

However, this functionality is not available in all countries. Thus, a specific procedure was built to calculate 
automatically the urban intersections using appropriately the DirectionsRequest API, i.e., by executing this API for identifying the path between any two urban streets, but discarding the results if the path consists of more than two steps. In this way, we can determine the intersection of two streets as the point interconnecting the two steps. Fig. 2 shows one of the intersections identified by the above procedure visualized on the relevant Google Map.

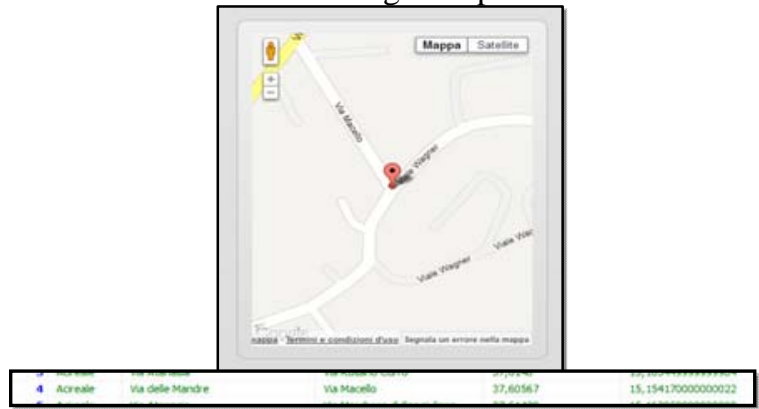

Figure 2. A node inside our database of intersections

In case an intersection cannot be calculated automatically, e.g., if the intersection cannot be identified by the road names of the Google Maps database, it is possible to put a marker related to this intersection directly on the Google Maps (see fig. 3) and to save its coordinates in the database.

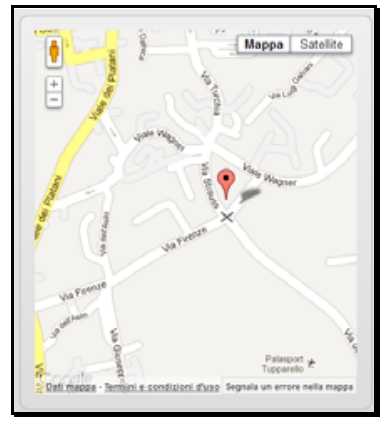

Figure 3. Calculating a node through the marker

Fig. 4 shows the Urban Road Graph of a small city built by our automated procedure. In the road graph of our monitoring system, each node is an intersection and any pair of adjacent intersections is featured by the travel time computed by using the GPS data received from various mobile devices.

Building the mentioned Urban Road Graph is a rather complex calculation that takes a long time; but it is executed very rarely, i.e., at the beginning of the monitoring service and when there is a change of the territory infrastructure, e.g., the creation of new roads or the elimination of an old one. Therefore the time performance of this calculation does not interfere with the time performance of the proposed monitoring service. After having built the Urban Road Graph, it is easy to follow the mobile users using the GPS data sent from their mobile devices at regular intervals to the MIS. Processing such data allows the MIS to localize the driver positions as shown in [3], and to monitor the traffic flows, as illustrated in the next section.

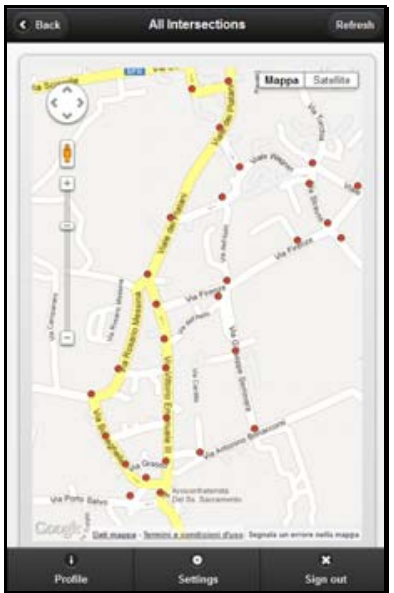

Figure 4. Urban Road Graph

\section{Monitoring TrafFic FlOWS FROM GPS SignALS}

As known, traffic flows may be measured by monitoring the car flows using pneumatic tubes or magnetic loops [4], traffic videos [5] even in environments with little luminosity [6] or affected by noisy conditions [7], and from traffic policemen perceptions [8].

This section points out how it may be provided by the GPS of the mobiles of the drivers with a method that is simpler than the ones proposed in literature, e.g., [9], but fast enough to allow the server to calculate the travel time for each road section in real time.

In this way it is possible to display the current traffic flows on the map of the driver mobile using a suitable chromatic scale, and to provide the possible decision support system running on the server, e.g., [10], or on the mobile [11] with relevant information to adjust the user planned activity.

The proposed methodology simply needs that the GPSs of the users traveling the urban road graph transmit at regular intervals to the server the user coordinates together with the time at which these coordinates have been detected. This allows the MIS to identify the driver positions in the urban road graph as shown in fig.5.

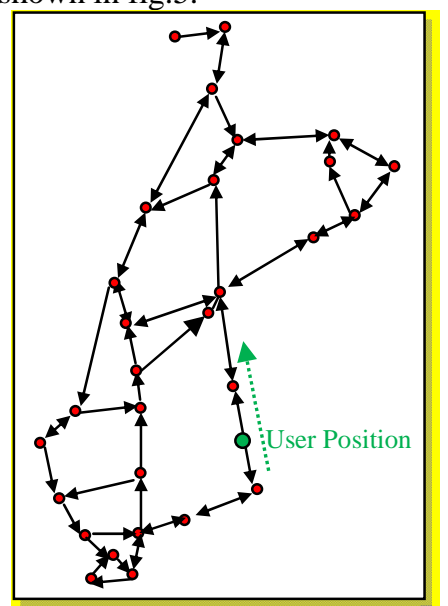

Figure 5. Any user sends information about his position while traveling the Urban Road Graph thus allowing the MIS to identify the position. 
Once obtained the GPS information from a suitable number of cars related to a given road, the server processes the stored data for this road to find the average speed, but before starting this procedure the traffic monitoring system manager is requested to use the Admin Control Panel (see fig. 6) to fix the chromatic scale to be used for differentiating the road traffic flows, and the maximum speed limit to be used as a threshold by the control algorithm to eliminate the incorrect data sent from the GPSs.

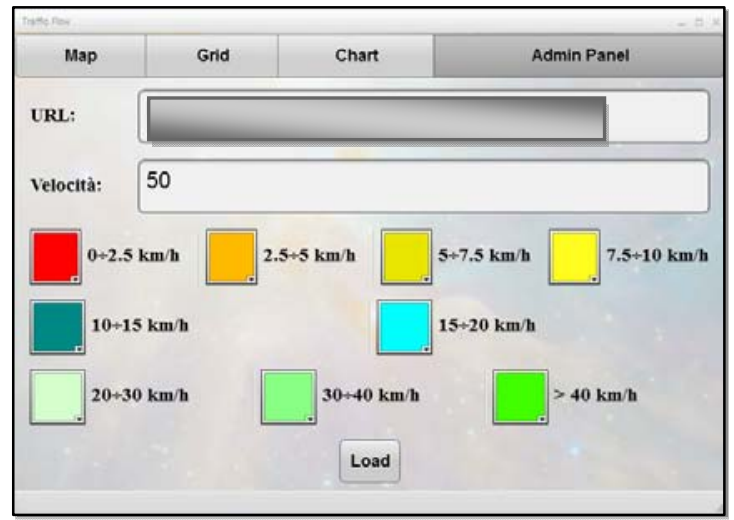

Figure 6. Admin Control Panel of the Traffic Monitoring software

After setting the color coding, the algorithm will proceed to calculate the speed of any car for every step between two consecutive measurements taken from the GPS signal. Averaging the information received for each road, the MIS is able to visualize the conditions of the road traffic according to the mentioned color scale, as shown in fig.7.

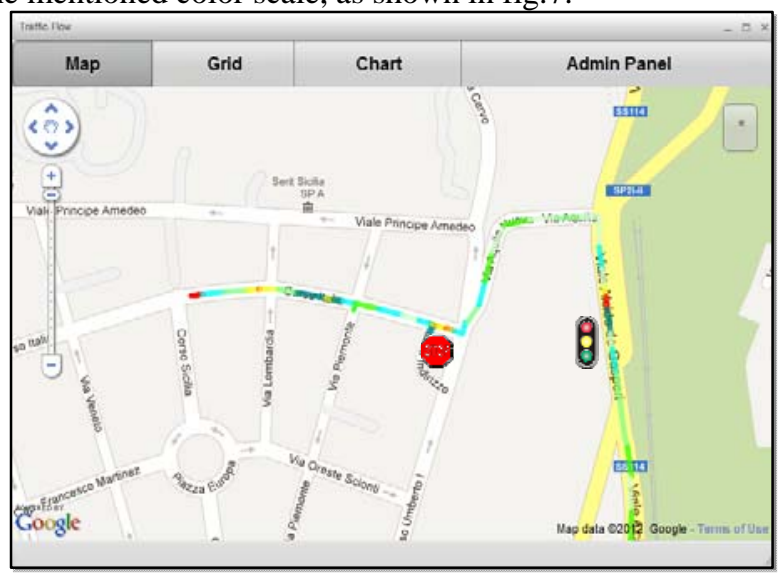

Figure 7. Road Traffic Flow conditions

A general inspection of the traffic flows of the peripheral road drawn in fig.7 demonstrates that the adopted monitoring system is accurate enough. In fact, the traffic conditions at the intersections are pointed out by a color, i.e., yellow, associated with a low car speed, whereas away from the intersections the traffic condition becomes more fluid as it is highlighted by the cyan color corresponding to a speed of about $15 \mathrm{~km} / \mathrm{h}$.

To provide the mobile user with more detailed information it might be useful to draw, for each road, two colored arcs related to the two different directions. Also, for each direction, it may be useful to differentiate the flows for each lane. The former problem could be solved by drawing for each road two different arcs depending on the car direction, whereas the latter may be solved representing, close the intersection, the different lane flows with different colors. This information could be provided at the user request.

Fig. 8 shows a possible representation of this solution, in which the condition for each traffic lane is drawn by an info window that specifies the conditions for each lane only if the user clicks the marker (or the traffic light) associated to the intersection. This representation is currently under test and will be illustrated more deeply in further works.

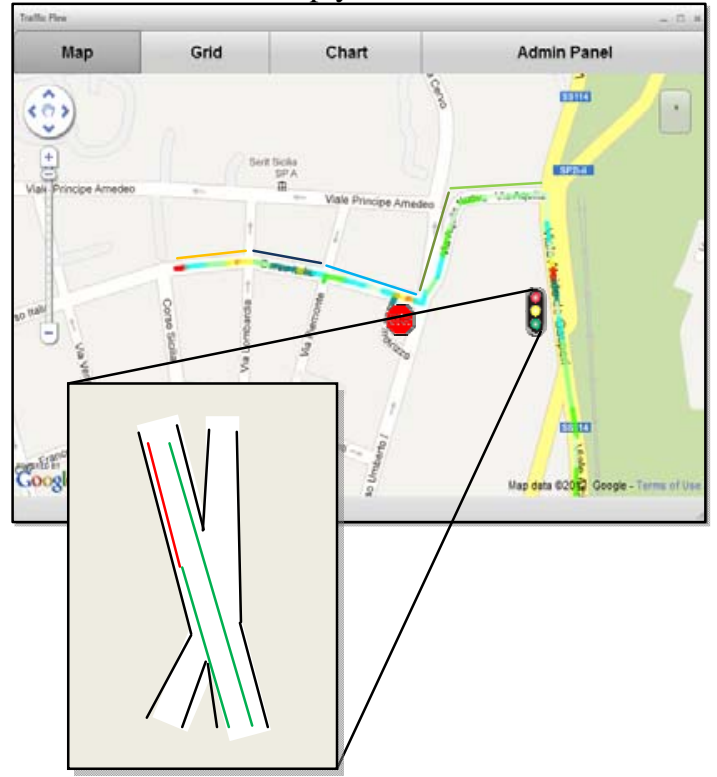

Figure 8. Representation of road lane flows close signalized intersections

\section{CASE STUDY}

This section considers the case of a commuter traveling daily from a small city to the main one of a metropolitan area, i.e., from Acireale to Catania. Usually there are few paths to choose, but information regarding the traffic flows allows the commuter to change the current path to a more fluid path, if any.

As above said, the proposed simple monitoring system is obtained by averaging the information sent from the mobiles of the interested commuters to the server. The server processes the GPS data received from each driver thus localizing each car in real time and improving the speed estimation of each road by adding a new value to the previously computed average (mobile average). Fig.9 shows the driver localization of a driver obtained by using the regular GPS signals sent by her/his mobile. High (low) marker density corresponds to low (medium) speed. 


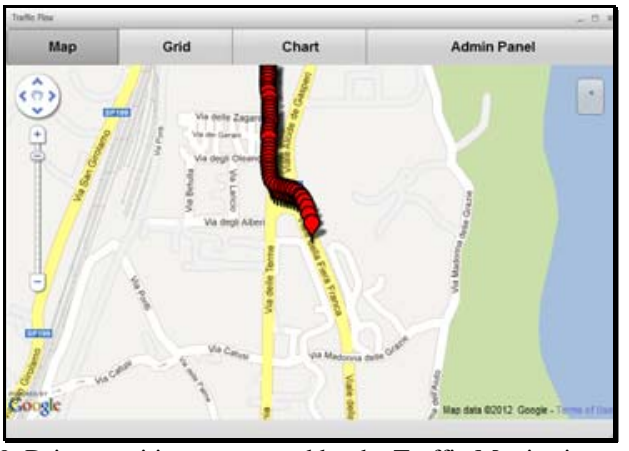

Figure 9. Driver positions computed by the Traffic Monitoring software

The server (or the driver mobile) re-computes at regular intervals the path to destination for each driver so that $\mathrm{s} / \mathrm{he}$ may be informed timely when a significant better path to destination is available. Let us note that the average speed of each segment is used to eliminate wrong measurements, e.g., if the software measures an excessive speed between two consecutive points with respect to the current average speed, then, with high probability, the measured coordinates of the last point are incorrect and, therefore, it is discarded as illustrated in fig. 10 where the point numbered 52 is discarded since the speed between the points 51 and 52 is too high.

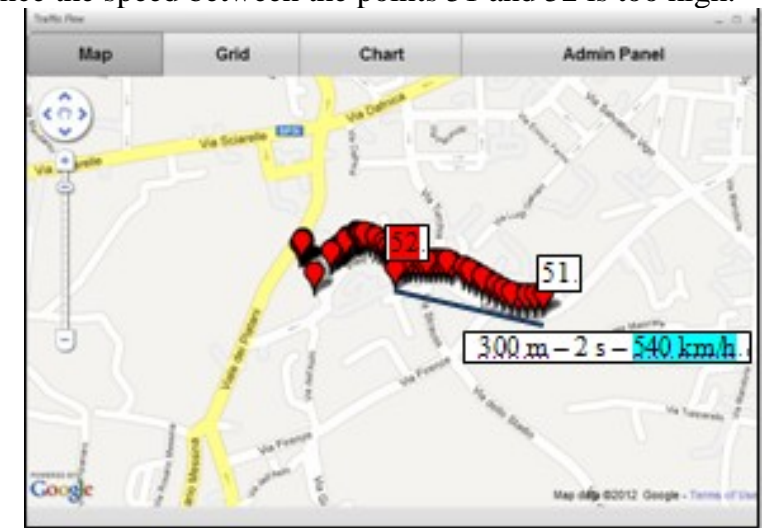

Figure 10. Point 52 is an error detection of the GPS

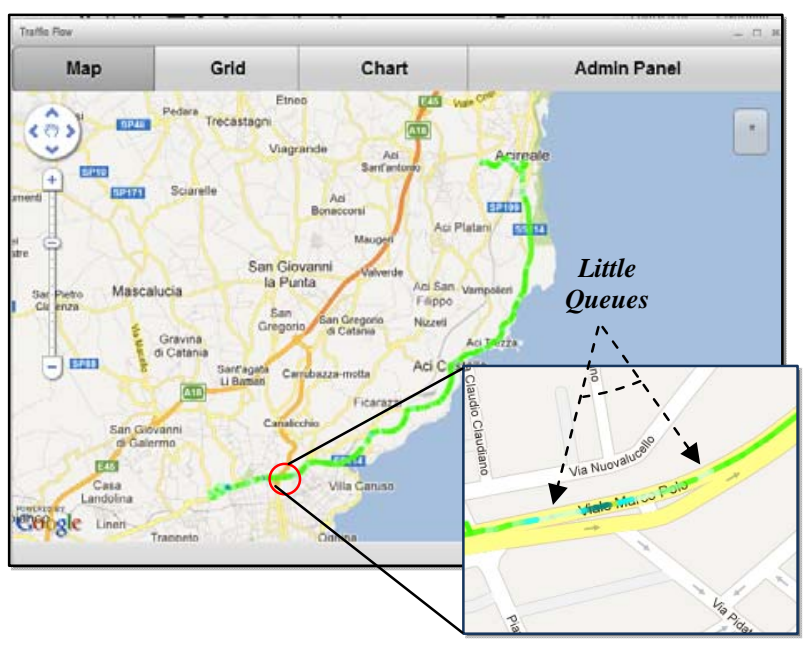

Figure 11. Traffic Flow Condition from Acireale to Catania with some little congestion close the main city.
Fig. 11 shows the final result of the proposed procedure: the traffic flow seems fluid for the main path followed by the commuters, but observing to a greater detail we may see some areas where the driver should behave more carefully since the traffic is a little congested.

\section{CONCLUDING REMARKS}

To build the traffic monitoring system it is necessary that enough drivers send their GPS data, during the travel, to the server to compute the traffic flows in real time. To encourage the users to do this, we plan to give them some discounts for receiving the traffic information and/or to provide them with some particular additional features.

Since it may be difficult to have traffic information from the driver GPSs for the entire traffic network, a mixed monitoring infrastructure seems more suitable to cover the entire network, e.g., traffic tubes/loops may be used in the downtown streets with little traffic, while webcams may be adopted in areas where it is useful to carry out video surveillance as well as to calculate the traffic flows. The proposed GPS monitoring system could be suitable for the periphery and for the downtown area with high traffic.

Currently the proposed monitoring system is under test within a mobility information platform called Wi-City [3], where the main server is implemented in Ruby on Rails [12] and users are informed by means of JQmobile scripts [13]. Also, a software has been developed using Flash Builder [14] to be implemented on the user mobiles to improve time performance [11]. Also a proper format according to some standard urban vocabulary (urban ontology), e.g., [15] and [16], to represent the colored maps and the alert information is planned to make available the basic monitoring system information to any driver mobile.

\section{REFERENCES}

[1] W. Shi, Q-J. Kong, Y. Liu, A GPS/GIS Integrated System for Urban Traffic Flow Analysis, Proc. of the 11th International IEEE Conference on Intelligent Transportation Systems Beijing, China, 2008.

[2] A. Faro, D. Giordano, C. Spampinato, Integrating Location Tracking, Traffic Monitoring and Semantics in a Layered ITS Architecture. Intelligent Transport Systems, vol.5(3), 197-206, 2011.

[3] A. Costanzo, A. Faro, D. Giordano, M. Venticinque, Wi-City: A federated architecture of metropolitan databases to support mobile users in real time, IEEE Proc. Int. Conf. on Computer \& Information Science (ICCIS), Vol.2, 586-591, 2012.

[4] G. Leduc, Road Traffic Data: Collection Methods and Applications, Working Papers on Energy, Transport and Climate Change, N.1, JRC European Commission, 47967, 2008.

[5] A. Crisafi, D. Giordano, C. Spampinato, GRIPLAB 1.0: Grid Image Processing Laboratory for Distributed Machine Vision Applications. Proc. Int. Workshop on Enabling Technologies: Infrastructure for Collaborative Enterprises, WETICE '08. 188-191, IEEE, 2008.

[6] A. Faro, D. Giordano, C. Spampinato, Adaptive background modeling integrated with luminosity sensors and occlusion processing for reliable vehicle detection. IEEE Transactions on Intelligent Transportation Systems, Vol.12(4), 1398 - 1411, 2011.

[7] F. Cannavò, G. Nunnari, D. Giordano, C. Spampinato, Variational Method for Image Denoising by Distributed Genetic Algorithms on GRID Environmentc. Workshops on Enabling Technologies: Infrastructure for Collaborative Enterprises, WETICE '06. 227-234, IEEE, 2006. 
[8] A. Faro, D. Giordano, C. Spampinato, Evaluation of the Traffic Parameters in a Metropolitan Area by Fusing Visual Perceptions and CNN Processing of Webcam Images, IEEE Transactions on Neural Networks, Vol. 19 (6), 1108-1129, 2008.

[9] S. Tao, V. Manolopoulos, S. Rodriguez, A. Rusu, Real Time Urban Traffic State Estimation with A-GPS Mobile Phones as Probes, Journal of Transportation Technologies, N.2, 22-31, 2012.

[10] V.L. Sauter, Decision Support Systems for business intelligence, Wiley, 2011.

[11] A. Costanzo, A. Faro, D. Giordano, Wi-City: living, deciding and planning using mobiles in Intelligent Cities , 3rd International Conference on Pervasive and Embedded Computing and Communication Systems, PECCS, Barcelona, INSTICC, 2013

[12] M. Hartl, Ruby on Rails 3, Addison Wesley, 2011.

[13] M. David, Developing Websites with jQueryMobile, Focal, 2011.

[14] M. Corlan, Flash Platform Tooling: Flash Builder, Adobe, 2009.

[15] A. Faro, D. Giordano, A. Musarra, Ontology Based Mobility Information Systems. Proc. of IEEE Systems, Men and Cybernetics SMC03, vol.3, 4288-4293, 2003.

[16] J. Zhai, J. Jiang, Y. Yu, J. Li, Ontology-based Integrated Information Platform for Digital City, IEEE Proc. of eWireless Communications, Networking and Mobile Comp., WiCOM '08, 2008. 